Document downloaded from:

http://hdl.handle.net/10251/155000

This paper must be cited as:

Zaffino, R.; Seimetz, M.; Quirión, D.; Ruiz-De La Cruz, A.; Sánchez, I.; Mur, P.; Benlliure, J.... (2018). Preparation and characterization of micro-nano engineered targets for highpower laser experiments. Microelectronic Engineering. 194:67-70.

https://doi.org/10.1016/j.mee.2018.03.011

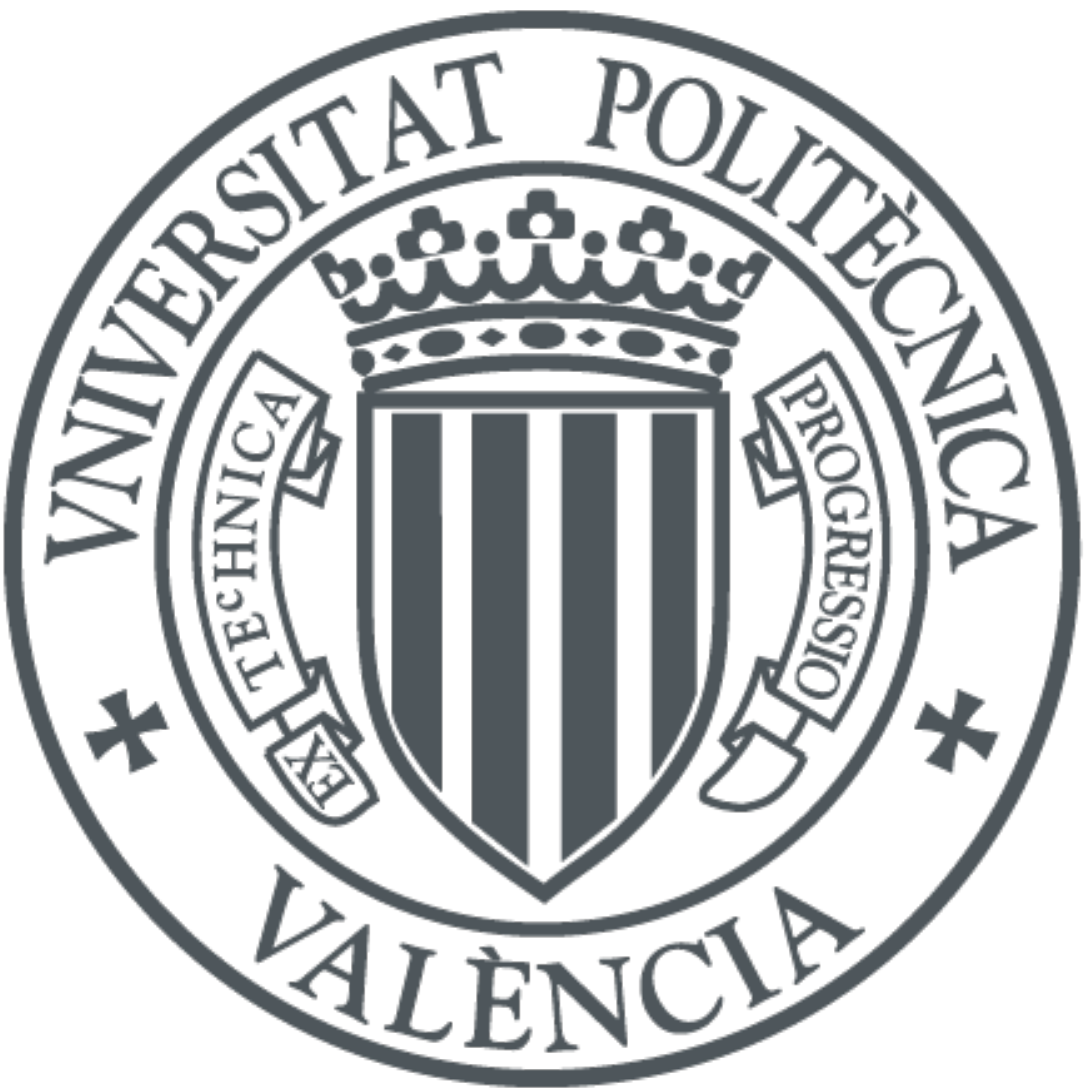

The final publication is available at

https://doi.org/10.1016/j.mee.2018.03.011

Copyright Elsevier

Additional Information 


\section{Preparation and characterization of micro-nano engineered targets for high-power laser experiments}

Rosaletizia Zaffino ${ }^{1}$, Michael Seimetz ${ }^{2}$, David Quirión ${ }^{1}$, Alejandro Ruiz de la Cruz ${ }^{3}$, Isabel Sánchez $^{3}$, Paula Mur², José Benlliure ${ }^{4}$, Lucia Martín ${ }^{4}$, L. Roso ${ }^{5}$, José María Benlloch², Manuel Lozano $^{1}$, Giulio Pellegrini ${ }^{1}$

1) Instituto de Microelectrónica de Barcelona IMB-CNM (CSIC) C/dels Til·lers Campus UAB, Cerdanyola del Vallès, Barcelona 08193, Spain

2) Instituto de Instrumentación para Imagen Molecular (I3M) CSIC Universitat Politècnica de València Camino de Vera s/n, Ed. 8B-N-1a, 46022 Valencia, Spain

3) Proton Laser Applications S.L., Av. Vilafranca del Penedés 11 a 08734 Olérdola (Barcelona), Spain

4) Dpto. de Física de Partículas, Campus Vida USC, Rua Xoaquín Días de Rábago S/N, E15782 (Santiago de Compostela), Spain

5) Centro de Láseres Pulsados (CLPU), C/ Adaja 8, 37185 Villamayor (Salamanca), Spain

E-mail: rossella.zaffino@imb-cnm.csic.es

The continuous development of ultra-fast high-power lasers (HPL) technology with the ability of working at unprecedented repetition rates, between 1 and $10 \mathrm{~Hz}$, is raising the target needs for experiments in the different areas of interest to the HPL community. Many target designs can be conceived according to specific scientific issues, however to guarantee manufacturing abilities that enable large number production and still allow for versatility in the design is the main barrier in the exploitation of these high repetition rate facilities. Here, we have applied MEMS based manufacturing processes for this purpose. In particular, we have focused on the fabrication and characterization of submicrometric conductive membranes embedded in a silicon frame. These kinds of solid targets are used for laser-driven particle acceleration through the so-called Target Normal Sheath Acceleration mechanism (TNSA). They were obtained by top-down fabrication alternating pattern transfer, atomic layer deposition, and selective material etching. The adaptability of the approach is then analyzed and discussed by evaluating different properties of targets 
for use in laser-driven particle acceleration experiments. These characteristics include the surface properties of membranes after fabrication and the high density of the target array. Finally, we were able to show their efficiency for laser-driven proton acceleration in a series of experiments with a 3 TW table-top laser facility, achieving stable proton acceleration up to $2 \mathrm{MeV}$.

\section{Introduction}

Modern lasers can deliver an enormous amount of energy (0.1-100 J) in a pulse of ultra-short duration ( $\approx 50 \mathrm{fs}$ ) focused on a very tiny target area, typically of a few microns diameter [1]. These extremely high intensities $\left(10^{18}-10^{20} \mathrm{~W} / \mathrm{cm}^{2}\right)$ allow for the study of novel plasma physics effects emerging from the exotic matter states created by laser-matter interaction and which are of interest to many areas of physical science, including laser and particle beam physics, material science, laboratory astrophysics, and high energy particle physics. The range of applications within these subjects is even wider and extends to the fast ignition approach for inertial confinement fusion [2], plasma density diagnostics [3], new sources of radiation through laser-particle driven acceleration schemes [4] with the potential to develop a new generation of particle injectors that may be suitable for isotope production [5], medical proton therapy [6]. The growing interest in ultra-intense laser-matter interactions and the increasing number of facilities available at national and international laboratories, prompt forward the research on target designs useful to achieve laser-plasma coupling regimes proper to the study of the different phenomena. Several target designs can be conceived according to each case study, ranging from gaseous to solid. The increasing repetition rate of HPL installations which could imply between hundreds and thousands of consumable samples per hour, urges the development of highthroughput, customizable, and cost-effective strategies to fully exploit the inherent possibilities [7].

MEMS based fabrication processes such as lithography, wet, dry, surface, bulk, and silicon micromachining, and thin film techniques could be used to fabricate from simple to complex target designs versatile either for low and high repetition rate experiments, given the scalability of these processes to large size production. Thin solid targets with thicknesses ranging between 1-30 $\mu \mathrm{m}$, such as plain foils of different materials, have been involved in laser-particle acceleration through TNSA since early 
experiments in the field [8]. In this laser-matter interaction regime, which is the dominating one at laser intensities of $10^{18}-10^{19} \mathrm{~W} / \mathrm{cm}^{2}$ [9], particle acceleration is produced by charge field separation and an intense electric field on the rear target surface arising from the generation of relativistic electrons by the laser pulse focused on the front side. The production of stable particle beams for applications in radiobiology, medicine, or material science requires high repetition rate working regimes, so mass production of targets for laser-driven particle acceleration with limited costs is essential to the development of the field. In this regard, the suitability of MEMS based manufacturing strategies for the production of simple and also complex targets for TNSA has been addressed previously. Picciotto et al. [10], [11] applied microfabrication techniques to optimize the structure and geometry of hydrogenated silicon membrane targets with a thickness of $(17 \pm 1) \mu \mathrm{m}$ which allowed for multi-MeV proton beam acceleration at the PALS facility in Prague. Methods from micro and nano-fabrication have been used to enhance the surface properties of standard plan foil targets. For example, in [12], [13] a monolayer of polystyrene beads of $400 \mathrm{~nm}$ diameter was assembled on the laser-irradiated side of a $500 \mathrm{~nm}$ Mylar foil resulting in increase of the proton beam homogeneity compared to flat targets, whereas when assembled on the rear target surface this resulted also in enhancement of the proton beam divergence which could be useful for some applications such as proton radiography. Here, we have explored top-down based fabrication in order to obtain aluminum, and gold-based, membranes with thicknesses below $1 \mu \mathrm{m}$ in a silicon frame which were then used as targets for laser driven particles acceleration experiments through the TNSA mechanism.

\section{Experiment preparation and setup}

\subsection{Target fabrication}

To obtain metallic membranes made of aluminum or gold with variable thicknesses we made use of the top-down fabrication approach according to the process flow schematized in Fig. 1 which includes the following principal steps: photolithography to transfer the mask pattern on the front side of double polished $<100>$ Si 4" wafers, followed by metallization of the wafers back side, and silicon micromachining on the front side to obtain free-standing membranes. Before mask pattern transfer through 
UV lithography, wafers were submitted to thermal oxidation for the growth of a variable thickness of $\mathrm{SiO}_{2}$, and to LPCVD for the deposition of a $\mathrm{Si}_{3} \mathrm{~N}_{4}$ layer of $(1810 \pm 24)$ $\AA$ A. Both are thereafter used as mask during Si etching. Back side metallization was achieved by sputtering aluminum, or in case of gold-based membranes by evaporating first an adhesion bi-layer composed of $20 \mathrm{~nm} \mathrm{Ti}$ and $30 \mathrm{~nm}$ Ni followed by the desired thickness of gold. Before proceeding with silicon micromachining on the wafer's front side, we spread out on the backside a generous wax layer (cured at $100^{\circ} \mathrm{C}$ during $1 \mathrm{~h}$ ) which can be easily removed after short soaking in toluene. This was done in order to protect the metallized side during next fabrication steps involving the use of two very strong acidic solutions which could threaten the membranes integrity. The wax layer resulted effective also to protect membranes during transport and further manipulations until their use in experiments. The intrinsic fragility of ultra-thin membranes has been, in fact, pointed out [7] as the main limitation to their use for laser-driven acceleration, however this simple trick drastically increases the number of useful membranes per wafer. Thereafter, silicon micromachining was accomplished by wet etching using a $40 \% \mathrm{KOH}$ solution at $80^{\circ} \mathrm{C}$. The etching rate of $<100>\mathrm{Si}$ for this bath concentration and temperature is $(60 \pm 5) \mu \mathrm{m} / \mathrm{h}$ and it took between 8 and 9 hours of processing to complete. This is followed by etching the underlying $\mathrm{SiO}_{2}$ by rapidly exposing the wafer to HF (dip HF). Both procedures are followed by abundant washing with cold water.

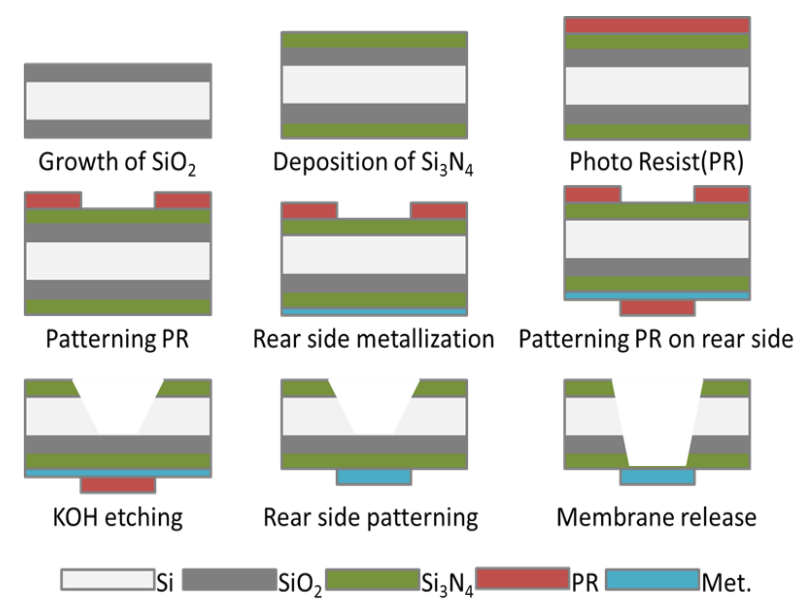

Fig. 1 Cross section of the process flow to fabricate a single membrane. 
Bulk Si micromachining has been largely applied in integrated microsystems and MEMS processing to achieve deep Si features that, as in our case, can extend through the entire wafer thickness. Wet, anisotropic etching of $\mathrm{Si}$ is extremely dependent on the crystallographic structure of the material. It relies, in fact, on the etching rates of the bath along planes with different orientations. $\mathrm{KOH}$ attacks preferentially in the $<100\rangle$ direction giving rise to characteristic $V$ shaped walls forming an angle of $54.7^{\circ}$ with the surface [14].

The design of membrane arrays for high power laser experiments has to fulfill several requirements. Arrays with high density of membranes are sought after because they allow for performing reliable experiments with reduced time and cost. The need for high array density though has to be balanced with those imposed by laser alignment and focusing, debris, and target fratricide. Below $1 \mathrm{~J}$ pulse energy, as in our case, neither debris contamination nor target fratricide are expected to impose major difficulties, so that the main constraint for our design was represented by the prerequisite for precise laser focusing and alignment. Whereas the laser spot has an area of $5 \times 11 \mu \mathrm{m}^{2}$ [15], membranes have been fabricated with a larger area of $1 \mathrm{~mm}^{2}$. This was the minimum area on which we could perform laser alignment and focusing according to the results of a preliminary test performed by using membranes with areas down to $0,01 \mathrm{~mm}^{2}$. Improvements in our alignment procedure to overcome this limitation are presently under study. Regarding this point, it should be also considered that damage propagation uses to extend beyond the size of the laser spot, as we could appreciate after analysis of shot targets that we will comment later.

Results of the fabrication are shown in Fig.2 (a)-(b) where the mask layout for one target cell is displayed together with its realization. 

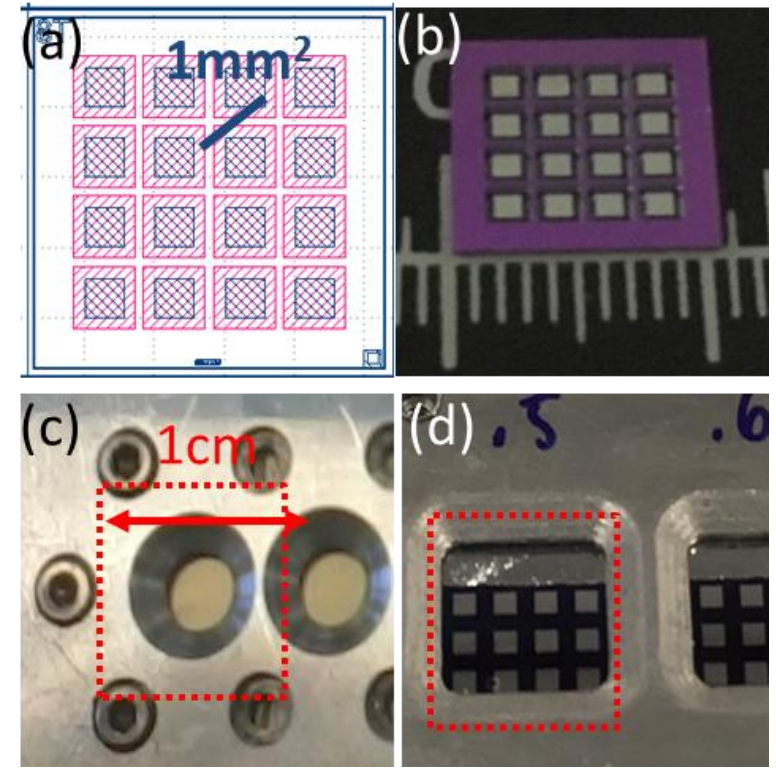

Fig.2 (a) Mask layout, (b) a target cell after fabrication, (c) Commercial foil and (d) MEMS membranes targets mounted on respective holder.

After fabrication the membranes surface shows several folds that are results of compressive stress in the film. These are unfavorable for laser alignment and focusing, being such operations much easier to realize in case of a flat (although rough) target surface, like in membranes characterized by tensile stress which are however more fragile. Also it should be mentioned that TNSA ions, as the acronym suggests, are released from the surface normal to the charge separation field, thus the membrane flatness is critical to control the beam direction. Residual stress is a common issue in the micro-fabrication of large area thin membranes on silicon substrates, and it has been deeply studied for its importance on the functionality of semiconductor based devices. Handling of strain in membranes fabricated on silicon substrates has received a lot of attention since early research [16]. Membranes are, in fact, largely used in MEMS and microelectronic devices and their role is often essential for the correct functioning of such equipments. Strain can be, indeed, used to tune material properties in membranes with nanometric thicknesses according to specific applications, as for example in case of silicon nitride membranes which have been recently used as nanomechanical resonators [17]. Balancing the stress of one material layer with the opposite one from another material layer has been widely explored in this field. In our case, this kind of strategy appears inadequate because it would result in the addition of a layer that should then be etched away thus increasing fabrication 
time. For this reason, in order to reduce compressive stress in membranes tethered on $\mathrm{SiO}_{2}$, we exposed membranes to a rapid annealing procedure $\left(40 \mathrm{~s}, 400^{\circ} \mathrm{C}\right)$ after that we could observe total release from compressive stress to tensile one. Alternatively, we could observe compressive stress release also in case of free-standing membranes after dip $\mathrm{HF}$ of $\mathrm{SiO}_{2}$. Lastly, from Fig. 2(c)-(d) it is possible to appreciate the high density of MEMS based membranes (12:1) which are available for laser interactions in the same space $\left(1 \mathrm{~cm}^{2}\right)$ occupied by a commercial foil mounted in the corresponding holder. This constitutes a clear advantage featured by MEMS based membranes with respect to the standard foils used as targets for HPL experiments, not only in regard of increasing the agility of tests but especially in view of the development of applications based on the TNSA mechanism for which a high repetition rate working regime is mandatory.

The fabrication process of membranes such as described here is a standard one which is well-known in the field of MEMS since early times. The last steps, $\mathrm{KOH}$ etching and dip HF, are the most critical ones and the overall yield of the process depends from their successful execution. In fact, we estimated that the production yield for aluminum membranes, calculated as the number of unbroken membranes out of all target cells, drops from 1 to 0.7 after $\mathrm{KOH}$ etching and to 0.5 after dip HF. In the case of gold-based membranes the yield at the end of the fabrication increases to 0.8 due to the higher strength of gold against chemical attack. For this reason, whenever there are not strict prescriptions to follow for the choice of membranes materials, it would be appropriate to consider a (nanometric) gold plating as a means to protect membranes and to increase production yields.

\subsection{Laser-proton acceleration experiments}

All the experiments were carried out on a table-top, 3 TW Ti:Sa laser system based on Chirped Pulsed Amplification (CPA) developed by Proton Laser Applications, S.L. In the present series this system centered on $800 \mathrm{~nm}$ delivered $280 \mathrm{~mJ}$ pulses $(175 \mathrm{~mJ}$ on target) with pulse duration of $55 \mathrm{fs}$. A He-Ne laser coupled into the same optical path of the Ti:Sa was used to monitor the transverse position of the target. Laser focusing on thin membranes was achieved by a $60^{\circ}$ off axis parabolic (OAP) mirror coated with 
gold. Pulses were focused at an incident angle of $30^{\circ}$ to avoid damage to the laser and detectors. Two kinds of detection methods were used for the characterization of accelerated particles. CR-39 plates, a passive material particularly useful for [18] the discrimination of protons and ions from electrons, were placed $100 \mathrm{~cm}$ behind the target holder, and a time-of-flight (TOF) detector based on a fast plastic scintillator, 227 $\mathrm{cm}$ behind the target, was used to give a prompt readout of experimental results[19]. Both detectors were mounted inside a vacuum tube (for further details of the experimental setup see [15]).

Laser driven proton acceleration experiments have been performed at low repetition rate between $0.1 \mathrm{~Hz}$ and $0.5 \mathrm{~Hz}$ exploiting the set-up described in [15]. Briefly, this can be considered as a three-stage system constituted by an injection (laser and compression chamber) followed by acceleration (interaction chamber) and particle characterization sections. Here, we restrict our attention to the interaction chamber where protons are accelerated from laser interaction with the target. Inside the chamber, a motorized target holder allows for movement in the three spatial directions with a precision of $2.5 \mu \mathrm{m}$. It is precisely aligned in order to assure that the horizontal displacement (from one target cell to the next) implies only negligible displacement along the laser axis. It can load up to 16 individual target cells for a total of 256 membranes available for experiments within one vacuum cycle. Laser alignment and focusing were operated as explained in [15] for one membrane of each individual target cell. Along the laser propagation direction the focal position was identified using the back-reflection pattern of a visible control laser, with a precision of $\pm 25 \mu \mathrm{m}$. For subsequent membranes the longitudinal position with respect to the laser focus was varied within a range of $\pm 100 \mu \mathrm{m}$ to study the effects of proton energy variation. Our data analysis indicates that the maximum proton energy suffers only weak variations, accounting for less than $10 \%$ of the maximum, within $\pm 50 \mu \mathrm{m}$ around the focus position. We could clearly detect proton tracks for all the membrane thicknesses used without appreciating systematic differences between the maximum protons energy averaged over several shots. Results obtained by using the same experimental setup with MEMS based metallic membranes and plain foils are compared in Fig. 3. Target thicknesses refer to the principal, metallic layer (aluminum or gold). Shots on membranes with or without residual $\mathrm{SiO}_{2}$ layer are summarized into the same data 
points. The maximum proton energy observed from plain foils is $1.8 \mathrm{MeV}$ at $0.65 \mu \mathrm{m}$. For thicknesses above $1 \mu \mathrm{m}$ proton energies are lower, in agreement with previous experiments on the same setup [15]. With MEMS manufactured targets between 70 $\mathrm{nm}$ and $1 \mu \mathrm{m}$ similar values have been observed. The fact that, in this interval, proton energies do not increase towards thinner targets has been observed previously [20].

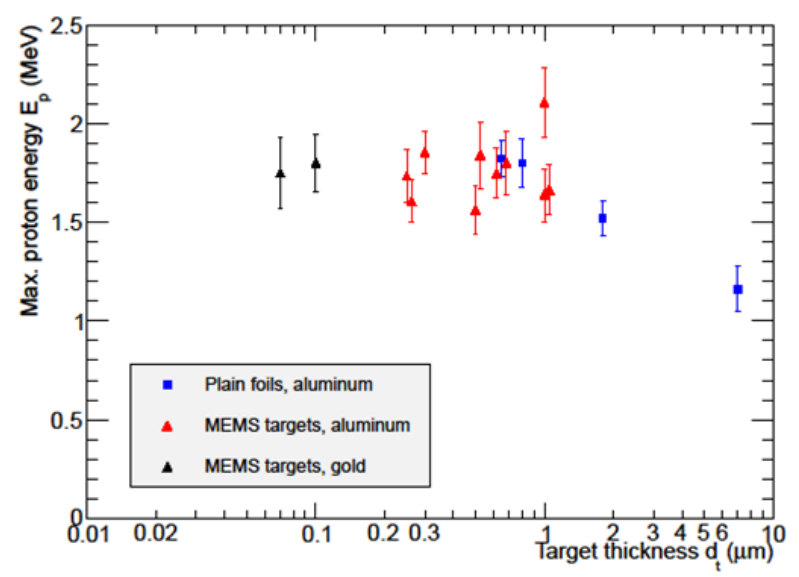

Fig. 3 Comparison between maximum proton energy achieved with standard, plain foils versus MEMS manufactured targets. Some data points, corresponding to different series of laser shots, have been slightly shifted horizontally for better visualization.

Finally, we would like to comment on the damage propagation observed on targets after the laser shots. Depending on the target material and focal position, circular or elliptic holes with several hundred $\mu \mathrm{m}$ diameters were formed, two orders of magnitude bigger than the laser focus, and some membranes were entirely destroyed. Nevertheless, the damage did not spread beyond the individual structures defined by the wafer. This result is particularly important in view of further miniaturization of our membranes ( $0.2 \mu \mathrm{m}$ lateral size) and the corresponding increase in array density. We are planning to corroborate these findings with smaller membranes.

\section{Conclusions.}

We made use of standard MEMS fabrication manufacturing to achieve wafer-scale fabrication of ultra-thin films used as targets for laser-driven particle acceleration. Thin film membranes were obtained by applying the common top-down fabrication approach and they were successfully used for laser-driven particle acceleration with a 
table-top, high-power laser. While their performance was found to be similar to standard, plain foils in terms of maximum proton energies, other advantages like the number of individual targets per area have become manifest. MEMS based fabrication techniques thus provide an efficient route for the high throughput production of targets for HPL experiments and applications.

\section{Acknowledgements}

The authors highly appreciate the collaboration of Radosys (Budapest) which provided CR39 detector material, etching bath, and readout equipment. This project has been financed by the Spanish Ministry for Economy and Competitiveness within the Retos-Colaboración 2015 initiative, ref. RTC-2015-3278-1. P. Mur has received a grant of the Garantía Juvenil 2015 program. This work has made use of the Spanish ICTS Network MICRONANOFABS partially supported by MEINCOM.

\section{References.}

[1] C. L. Haefner, A. Bayramian, S. Betts, R. Bopp, S. Buck, J. Cupal, M. Drouin, A. Erlandson, J. Horaék, J. Horner, J. Jarboe, K. Kasl, D. Kim, E. Koh, L. Koubíková, W. Maranville, C. Marshall, D. Mason, J. Menapace, P. Miller, P. Mazurek, A. Naylon, J. Novák, D. Peceli, P. Rosso, K. Schaffers, E. Sistrunk, D. Smith, T. Spinka, J. Stanley, R. Steele, C. Stolz, T. Suratwala, S. Telford, J. Thoma, D. Vanblarcom, J. Weiss, and P. Wegner, "High average power, diode pumped petawatt laser systems: A new generation of lasers enabling precision science and commercial applications," Proc. SPIE - Int. Soc. Opt. Eng., vol. 10241, 2017.

[2] R. Betti and O. A. Hurricane, "Inertial-confinement fusion with lasers," Nat. Phys., 2016.

[3] L. B. Da Silva, T. W. Barbee, R. Cauble, P. Celliers, J. Harder, H. R. Lee, R. A. London, D. L. Matthews, S. Mrowka, J. C. Moreno, D. Ress, J. E. Trebes, A. Wan, and F. Weber, "X-ray lasers for high density plasma diagnostics (invited)," Rev. Sci. Instrum., vol. 66, no. 1, pp. 574-578, 1995.

[4] M. Borghesi, "Laser-driven ion acceleration: State of the art and emerging mechanisms," Nucl. Instruments Methods Phys. Res. Sect. A Accel. Spectrometers, Detect. Assoc. Equip., vol. 740, pp. 6-9, 2014.

[5] M. Seimetz, P. Bellido, F. Sanchéz, R. Lera, A. Ruiz de la Cruz, S. Torres-Peiró, L. Roso, A. Aguilar, P. Conde, A.J. Gonzales, A. Iborra, L. Moliner, J. P. Rigla, M. J. 
Rodriguez-Alvarez, S. Sanchez, A. Soriano, and J. M. Benlloch "Detailed requirements for a laser-based proton/ion accelerator for radioisotope production" 2015 IEEE Nuclear Science Symposium and Medical Imaging Conference (NSS/MIC), ISBN 9781467398626.

[6] V. Malka, S. Fritzler, E. Lefebvre, E. D'Humièrs, R. Ferrand, G. Grillon, C. Albaret "Practicability of proton therapy using compact laser systems" Medical Physics, 31 (6) 1587-1592 (2004).

[7] I. Prencipe, J. Fuchs, S. Pascarelli, D. W. Schumacher, R. B. Stephens, N. B. Alexander, R. Briggs, M. Büscher, M. O. Cernaianu, A. Choukourov, M. De Marco, A. Erbe, J. Fassbender, G. Fiquet, P. Fitzsimmons, C. Gheorghiu, J. Hund, L. G. Huang, M. Harmand, N. J. Hartley, A. Irman, T. Kluge, Z. Konopkova, S. Kraft, D. Kraus, V. Leca, D. Margarone, J. Metzkes, K. Nagai, W. Nazarov, P. Lutoslawski, D. Papp, M. Passoni, A. Pelka, J. P. Perin, J. Schulz, M. Smid, C. Spindloe, S. Steinke, R. Torchio, C. Vass, T. Wiste, R. Zaffino, K. Zeil, T. Tschentscher, U. Schramm, and T. E. Cowan, "Targets for high repetition rate laser facilities: needs, challenges and perspectives," High Power Laser Sci. Eng., vol 5, e17, 2017.

[8] S. C. Wilks, A. B. Langdon, T. E. Cowan, M. Roth, M. Singh, S. Hatchett, M. H. Key, D. Pennington, A. MacKinnon, and R. A. Snavely, "Energetic proton generation in ultra-intense laser-solid interactions," Phys. Plasmas Phys. Plasmas, vol. 8, no. 8, 2001.

[9] E. D’Humières, J. Fuchs, P. Antici, P. Audebert, E. Brambrink, E. Lefebvre, V. Malka, P. Mora, and Y. Sentoku, "PIC simulations of proton acceleration with high intensity lasers: The transparency regime, and interaction with underdense targets," in AIP Conference Proceedings, CP877, Advanced Accelerator Concepts: $12^{\text {th }}$ Workshop, 2006.

[10] A. Picciotto, D. Margarone, M. Crivellari, P. Bellutti, S. Colpo, L. Torrisi, J. Krasa, A. Velhyan, and J. Ullschmied, "Microfabrication of silicon hydrogenated thin targets for multi-MeV laser-driven proton acceleration," Appl. Phys. Express, vol. 4, no. 12, 2011.

[11] A. Picciotto, D. Margarone, J. Krasa, a. Velyhan, E. Serra, P. Bellutti, G. Scarduelli, L. Calliari, E. Krousky, B. Rus, and M. Dapor, "Laser-driven acceleration of protons from hydrogenated annealed silicon targets," EPL (Europhysics Lett., vol. 92, no. 
3, p. 34008, 2010.

[12] L. Giuffrida, K. Svensson, J. Psikal, D. Margarone, P. Lutoslawski, V. Scuderi, G. Milluzzo, J. Kaufman, T. Wiste, M. Dalui, H. Ekerfelt, I. G. Gonzalez, O. Lundh, A. Persson, A. Picciotto, M. Crivellari, A. Bagolini, P. Bellutti, J. Magnusson, A. Gonoskov, L. Klimsa, J. Kopecek, T. Lastovicka, G. A. P. Cirrone, C. G. Wahlström, and G. Korn, "Nano and micro structured targets to modulate the spatial profile of laser driven proton beams," J. Instrum., vol. 12, no. 3, 2017.

[13] L. Giuffrida, K. Svensson, J. Psikal, M. Dalui, H. Ekerfelt, I. Gallardo Gonzalez, O. Lundh, A. Persson, P. Lutoslawski, V. Scuderi, J. Kaufman, T. Wiste, T. Lastovicka, A. Picciotto, A. Bagolini, M. Crivellari, P. Bellutti, G. Milluzzo, G. A. P. Cirrone, J. Magnusson, A. Gonoskov, G. Korn, C. G. Wahlström, and D. Margarone, "Manipulation of laser-accelerated proton beam profiles by nanostructured and microstructured targets," Phys. Rev. Accel. Beams, vol. 20, no. 8, pp. 1-8, 2017.

[14] W. K. Schomburg, "Introduction to Microsystem Design," in Introduction to microsystem design, vol. 1, Springer, 2011, pp. 9-21.

[15] P. Bellido, R. Lera, M. Seimetz, A. Ruiz-De La Cruz, S. Torres-Peirò, M. Galán, P. Mur, I. Sánchez, R. Zaffino, L. Vidal, A. Soriano, S. Sánchez, F. Sánchez, M. J. Rodríguez-Álvarez, J. P. Rigla, L. Moliner, A. Iborra, L. Hernández, D. Grau-Ruiz, A. J. González, J. J. García-Garrigos, E. Díaz-Caballero, P. Conde, A. Aguilar, L. Roso, and J. M. Benlloch, "Characterization of protons accelerated from a 3 TW tabletop laser system .," JINST.,12, p. T05001, 2017.

[16] D. Jaeggi, "Thermal Converters by CMOS technology," PhD thesis, ETH Zurich, 1996.

[17] N. Luhmann, A. Jachimowicz, J. Schalko, P. Sadeghi, M. Sauer, A. Foelske-Schmitz, and S. Schmid, "Effect of oxygen plasma on nanomechanical silicon nitride resonators," Appl. Phys. Lett., vol. 111, no. 6, 2017.

[18] M. Seimetz, P. Bellido, P. García, P. Mur, A. Iborra, A. Soriano, E. Hülber, T. Hülber, J. García López, M.C. Jiménez-Ramos, R. Lera, A. Ruiz-de la Cruz, I. Sánchez, R. Zaffino, L. Roso, and J.M. Benlloch " Spectral characterisation of laseraccelerated protons with CR-39 nuclear track detector, Review of Scientific Instruments 89, 023302 (2018).

[19] M. Seimetz, P. Bellido, A. Soriano, J. G. López, M. C. Jiménez-Ramos, B. Fernández, 
P. Conde, E. Crespo, A. J. González, L. Hernández, A. Iborra, L. Moliner, J. P. Rigla, M. J. Rodríguez- Alvarez, F. Sánchez, S. Sánchez, L. F. Vidal, and J. M. Benlloch, "Calibration and performance tests of detectors for laser- accelerated protons," IEEE Transactions on Nuclear Science 62, 3216-3224 (2015).

[20] F. Dollar, S. A. Reed, T. Matsuoka, S. S. Bulanov, V. Chvykov, G. Kalintchenko, C. McGuffey, P. Rousseau, A. G. R. Thomas, L. Willingale, V. Yanovsky, D. W. Litzenberg, K. Krushelnick, and A. Maksimchuk, "High-intensity laser-driven proton acceleration enhancement from hydrogen containing ultrathin targets," Appl. Phys. Lett., vol. 103, no. 14, 2013. 